\title{
DETERMINISTIC STRESS MODELING FOR MULTISTAGE COMPRESSOR FLOWFIELDS
}

\author{
Stefan Stollenwerk \\ Institute of Propulsion Technology \\ German Aerospace Center (DLR) \\ Linder Hoehe, 51147 Cologne \\ Email: stefan.stollenwerk@dlr.de
}

\author{
Edmund Kügeler \\ Institute of Propulsion Technology \\ German Aerospace Center (DLR) \\ Linder Hoehe, 51147 Cologne \\ Email: edmund.kuegeler@dlr.de
}

\begin{abstract}
Unsteadiness is one of the main characteristics in turbomachinery flows. Local unsteady changes in static pressure must exist within a turbo-machine in order for that machine to exchange energy with the fluid. The primary reason for unsteady effects lies in the interaction between moving and stationary blade rows. The industrial design process of aero-engines and gas turbines is still based on Reynolds-averaged Navier-Stokes (RANS) techniques where the coupling of blade rows is carried out by mixing-planes. However, this methodology does not cover deterministic unsteadiness in an adequate way. For standard aero-optimization, detailed unsteadiness is not essential to the designer of turbomachines but rather its effect on the time averaged solution. The time averaged deterministic unsteadiness can be expressed in terms of deterministic stresses. The present paper presents two different modeling strategies for deterministic stresses that constitute an improvement of the conventional steady mixing-plane approach. Whilst one of the presented models operates with deterministic flux terms based on preliminary unsteady simulations, the other one, a novel transport model for deterministic stress, is a stand-alone approach based on empirical correlations and a wide range of numerical experiments. A 4.5 stage transonic compressor is analyzed regarding blade row interaction effects and their impact on the time averaged solution. The two models are applied to the compressor and their solutions are compared to conventional mixing-plane, time accurate and experimental data. The results for the speedline, the wake shapes, the radial distributions and the rotor blade loadings show that the deterministic stress models strongly improve
\end{abstract}

the RANS solution towards the time accurate and the experimental methods.

\section{NOMENCLATURE}

$a$ speed of sound

ADP aerodynamic design point

$C_{p}$ static pressure coefficient

DF deterministic fluxes

DS deterministic stresses

F flux vector

$L$ pitch

$\dot{m}$ mass flow rate

$M a$ Mach number

$p$ pressure

Q state vector

$r$ radius

RANS Reynolds-averaged Navier-Stokes

Re Reynolds number

$S$ source term

$t$ time

$u$ velocity component

URANS unsteady Reynolds-averaged Navier-Stokes

$x$ Cartesian coordinate

$y^{+}$non-dimensional wall distance

$\alpha$ flow angle

$\delta_{1} \quad$ displacement thickness

$\rho$ density

$\theta$ circumferential position 
$\mu \quad$ viscosity

$\xi$ curvilinear coordinate

$\Pi$ pressure ratio

$\tau$ shear stress

$\chi$ streamline curvature

$\Omega$ rotational speed

$\overline{(.)}$ averaged value

$(.)^{\prime}$ stochastic fluctuation

(.) $)^{\prime \prime}$ deterministic fluctuation

\section{Subscripts and Superscripts}

$c$ convective

$d$ deterministic

$i, j$ tensor indices

is isentropic

$L E$ leading edge

$m$ molecular

$R$ Rotor

red reduced

rel relative

$t$ turbulent

tan tangential

tot total

$v$ diffusive

\section{INTRODUCTION}

Turbomachinery flow fields contain various complex phenomena. The flow may be compressible, subsonic, transonic or supersonic and all properties can occur in a small geometric region. However, unsteadiness is the major characteristic in turbomachinery. Greitzer et al. [1] discern three regimes depending on their length scales: Small scale, associated with turbulence and transition, medium scale, related to the passage flow structure, i.e. phenomena on the order of the pitchwise blade spacing and large scale phenomena with length scales larger than the blade passage and include flutter, forced vibration or rotating stall. It has to be noted that these three scales of unsteadiness cannot always be clearly distinguished from each other and a spectral overlap may occur. The small scale unsteadiness has random or stochastic properties. In contrast to the stochastic or turbulent aspects deterministic phenomena of larger scale can be identified. The deterministic phenomena may be uncorrelated with the rotational speed of the engine (e.g. von Kármán vortices) or correlated with it. Blade row interaction during the stable operation of the machine is an example of this type, whereas rotating stall is an example regarding unstable operating conditions [2]. Some of the most important effects regarding blade row interaction are unsteady separation due to wakes passing over downstream blade rows and potential interactions between fixed and rotating blade rows. Unsteadiness can have a significant impact on the physics of the flow field and therefore on operating performance.

For a detailed representation of the unsteady effects, intensive time dependent simulations have to be applied. Steady state flow simulations are the commonly used design methods for modern aero-engines and gas turbines. These approaches operate in a steady mixing-plane like solution environment and do not cover the deterministic unsteadiness in an adequate way. For standard aero-optimization, detailed knowledge of the time-dependent phenomena is not essential to the designer of turbomachines but rather its effect on the time averaged solution. The time averaged deterministic unsteadiness can be expressed in terms of deterministic stresses [3]. Deterministic stresses are analoguous to turbulent stresses representing correlations between fluctuating flow quantities. The time scales of the deterministic motion (as mentioned above) are typically much larger than the time scales of the stochastic (turbulent) motion. If the deterministic stresses can be correctly integrated in a steady-state simulation, it would theoretically yield the same results as the time average of an unsteady solution.

There exist various approaches to include deterministic stresses in steady state simulations. First of all the classical modelling, where the deterministic stresses are directly computed and included in a RANS framework. Hall [4] developed an algebraic model describing the influence of an upstream wake on a downstream blade row based on spatial-temporal correlations. Rhie et al. [5] utilise a similar assumption with an additional interface treatment for which overlapping meshes are needed. Van de Wall et al. [6], as well as Charbonnier and Leboeuf [7] integrated additional transport equations in a RANS solver in order to model the deterministic stress tensor. Therefore the solution of six additional equations is needed for a three dimensional simulation. Another modeling class can be denoted as frequencydomain methods. A harmonic balance solver [8] operates in the frequency domain whereas the nonlinear harmonic technique [9] couples a nonlinear RANS method with a linearized approach. Finally, lumped deterministic stresses can be deduced from unsteady computations as proposed by Sondak et al. [10]. Lukovic et al. [11] recommended a neural network model for deterministic source terms, which would require a large database of unsteady calculations.

In this paper two different modeling startegies are proposed. The deterministic flux model [12] is akin to the Lumped Deterministic Stress approach but the deterministic contributions are expressed as fluxes rather than being lumped together in form of source terms. Even though both procedures are mathematically identical, the deterministic flux method turned out to provide improved numerical stability and accuracy. Furthermore it yields more information about the behavior of deterministic stress and its production and reveals its possible anisotropic properties. The transport model [13] is a classical approach which focuses on the modeling of potential and shock interaction. It will be shown later that this is the driving factor for unsteady interaction in the 
chosen test vehicle. However, within this approach the deterministic stresses are approximated by a Boussinesq assumption. The behaviour and distribution of the supplemental stress is described by a transport mechanism and integrated in the Navier-Stokes equations by means of an additional source term.

\section{NUMERICAL METHOD}

All simulations within this study were carried out with the three dimensional Navier-Stokes solver TRACE. The code has been developed at DLR's Institute of Propulsion Technology and is optimised for turbomachinery flows. The Navier-Stokes equations are discretized using the finite volume methodology. Roe's upwind scheme is employed to discretise the convective fluxes and central differences are used for the derivatives of the viscous fluxes. The solver handles structured, unstructured and hybrid multiblock grids. The implicit time-integration scheme allows large CFL numbers and leads to a fast convergence rate. Turbulence modeling is effected by a k- $\omega$ two-equation approach with turbomachinery-specific extensions. Several additional modules extend the solvers applicability to aeroelastic, aeroacoustic, multi-phase and real gas problems. Further information regarding the simulation system is provided in [14].

\section{Deterministic stress modeling}

Within the conventional Reynolds methodology, velocities are decomposed into mean and fluctuating components. In the theory of deterministic stresses (Adamczyk [3]), the fluctuating components are considered to have a turbulent and a deterministic part:

$$
u_{i}=\overline{u_{i}}+u_{i}^{\prime}+u_{i}^{\prime \prime}
$$

$\overline{u_{i}}$ represents the mean velocity that is averaged over all time scales, $u_{i}^{\prime}$ is the turbulent part and $u_{i}^{\prime \prime}$ the deterministic contribution. A consecutive application of a mass-weighted averaging and a Reynolds averaging leads finally to the total stress tensor:

$$
\tau_{i j}=\tau_{i j}^{m}+\tau_{i j}^{t}+\tau_{i j}^{d}
$$

$\tau_{i j}^{m}$ describes the molecular stress, $\tau_{i j}^{t}$ is the turbulent stress and $\tau_{i j}^{d}$ represents the deterministic stress. A detailed derivation of this expression can be found in [15].

Transport model for deterministic stress. The basic idea for the modeling of the deterministic stresses is to corre- late them with the local strain rate:

$$
\tau_{i j}^{d}=\overline{\rho u_{i}^{\prime \prime} u_{j}^{\prime \prime}}=\mu_{d}\left(\frac{\partial u_{i}}{\partial x_{j}}+\frac{\partial u_{j}}{\partial x_{i}}\right)
$$

As the strain rate is known in a steady simulation, only $\mu_{d}$ has to be modeled in order to calculate the deterministic stresses. This is effected by a scalar transport equation:

$$
\bar{\rho} \frac{\partial \mu_{d}}{\partial t}+\left(\bar{\rho} \overline{u_{i}}\right) \frac{\partial \mu_{d}}{\partial x_{i}}-\frac{\partial}{\partial x_{i}}\left[\left(\mu_{m}+\mu_{d}\right) \frac{\partial \mu_{d}}{\partial x_{i}}\right]=S_{\mu_{d}}
$$

The source term in this equation is a function of the streamline curvature and an axial distribution:

$$
S_{\mu_{d}}=f(\chi) \cdot \exp \left[\frac{2 \pi}{L} \sqrt{1-\left(\frac{\Omega r}{a}\right)^{2}} \cdot\left(x-x_{R_{L E}}\right)\right]
$$

The streamline curvature is calculated by the local derivation of the tangential unit vector in flow direction, a detailed derivation of the whole expression is provided in [13]. All quantities in the presented formulation are known within the mixing plane approach. In order to close the equation system, only equation (4) has to be solved additionally.

Deterministic flux model. Applying the total stress decomposition (2) to the Navier-Stokes equations, the diffusive flux terms can be split up in an analogous way and the equations read in flux vector formulation:

$$
\frac{\partial \mathbf{Q}}{\partial t}+\frac{\partial \mathbf{F}_{c, i}(\mathbf{Q})}{\partial \xi_{i}}-\operatorname{Re}^{-1} \frac{\partial}{\partial \xi_{i}}\left[\mathbf{F}_{v, i}^{m}(\mathbf{Q})+\mathbf{F}_{v, i}^{t}(\mathbf{Q})+\mathbf{F}_{v, i}^{d}\right]=0
$$

According to equation 2 the diffusive flux also consists of a molecular $\left(\mathbf{F}_{v, i}^{m}\right)$, a turbulent $\left(\mathbf{F}_{v, i}^{t}\right)$ and a deterministic part $\left(\mathbf{F}_{v, i}^{d}\right)$. The deterministic flux terms can be expressed as the difference between the total flux components of a time averaged unsteady solution and the total flux components of a steady solution [12]:

$$
\begin{aligned}
\mathbf{F}_{v, i}^{d} & =\operatorname{Re} \cdot \mathbf{F}_{c, i}\left(\overline{\mathbf{Q}_{\mathrm{URANS}}}\right)-\mathbf{F}_{v, i}^{m}\left(\overline{\mathbf{Q}_{\mathrm{URANS}}}\right)-\mathbf{F}_{v, i}^{t}\left(\overline{\mathbf{Q}_{\mathrm{URANS}}}\right) \\
& -\left[\operatorname{Re} \cdot \mathbf{F}_{c, i}\left(\mathbf{Q}_{\mathrm{RANS}}\right)-\mathbf{F}_{v, i}^{m}\left(\mathbf{Q}_{\mathrm{RANS}}\right)-\mathbf{F}_{v, i}^{t}\left(\mathbf{Q}_{\mathrm{RANS}}\right)\right]
\end{aligned}
$$

Within this work, the deterministic flux terms were computed separately for each operating point, so any DF computation needed a preliminary URANS solution. However, this is not absolutely necessary. The number of preliminary URANS computations can be reduced significantly in order to predict a whole 
speedline [12].

Both models were implemented, tested and calibrated within TRACE.

\section{TESTCASE}

The research compressor RIG 250 is a transonic axial compressor. Its front stages are typical of a low pressure compressor in modern aero-engines. It consists of four stages with a total pressure ratio of $\Pi_{t o t}=4.83$ at a reduced mass flow rate of $\dot{m}_{\text {red }}=46.3 \mathrm{~kg} / \mathrm{s}$ at the design point. The rotor tips of the first two stages are in the transonic regime, with relative rotor tip mach numbers of $M a_{\text {rel }} \approx 1.50$ for rotor 1 and $M a_{\text {rel }} \approx 1.45$ for rotor 2 , while the last two stages are entirely subsonic. The vanes of the first two stages, as well as the inlet guide vane, are adjustable, whereas the last two stators are cantilevered (Röber et al. [16]). An overview of the compressor is given in Fig. 1 and Tab. 1.

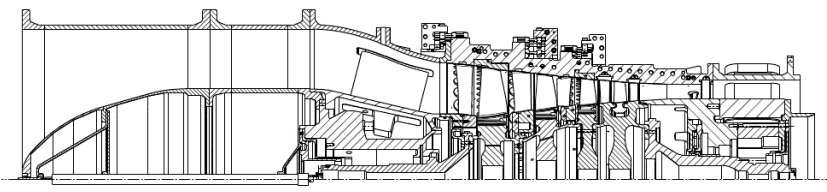

FIGURE 1. SKETCH OF THE 4.5 STAGE RESEARCH COMPRESSOR RIG 250.

TABLE 1. BLADE COUNTS AND MESH RESOLUTION FOR RIG 250

\begin{tabular}{llcr}
\hline Stage & Row & Passages & Gridpoints \\
\hline \multirow{2}{*}{1} & IGV \& swan neck & 40 & 1251012 \\
\hline & Rotor 1 & 23 & 869769 \\
& Stator 1 & 36 & 1004571 \\
\hline 2 & Rotor 2 & 28 & 882763 \\
& Stator 2 & 48 & 1014178 \\
\hline 3 & Rotor 3 & 38 & 882763 \\
& Stator 3 & 68 & 942477 \\
\hline 4 & Rotor 4 & 47 & 882763 \\
& Stator 4 \& exit duct & 80 & 1038618 \\
\hline
\end{tabular}

\section{Experimental Setup}

The experimental investigations were conducted at DLR's compressor test facility in Cologne. The facility provides a 10 MW electric motor drive and a flexible gearbox system. The drive shaft rotates at $20000 \mathrm{rpm}$. The rig test was carried out in an open loop configuration, meaning that ambient air was sucked through a tower into the settling chamber. The air passed through the rig via an air meter and was throttled by a ring throttle at the rear. For the aerodynamic investigation of the compressor a high level of instrumentation was installed (Johann and Heinichen [17]):

- mass flow measurements at the inlet of the rig,

- stator leading edge instrumentation for pressure and temperature,

- static pressure measurements,

- exit temperature and pressure rakes,

- hot film measurements on the stators

\section{Numerical Setup}

For the numerical analysis, the compressor was meshed using the standard TRACE preprocessing chain for structured grids [18]. In radial direction the mesh resolution was 75 nodes. The tip gaps over the rotors, the half-clearances over and below the adjustable guide vanes, as well as the clearances below the cantilevered stators, were each meshed using eleven nodes in the radial direction. For all blade rows, the meshes have an $\mathrm{OCH}$-topology, the clearances were meshed using H-blocks. The whole computational grid contains 8768914 points. Figure 2 shows a S1-plane exemplarily for the IGV and the first stage. On

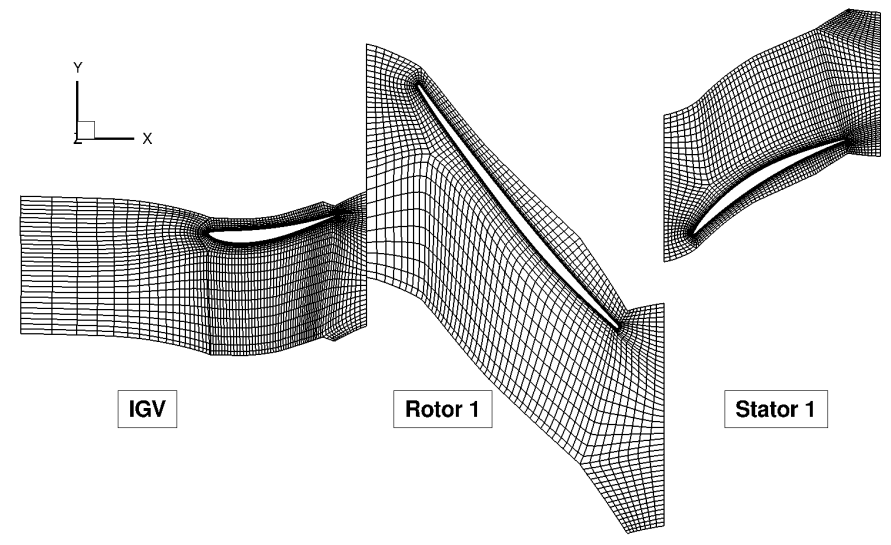

FIGURE 2. S1-PLANE OF THE COMPUTATIONAL GRID OF IGV AND FIRST STAGE AT 50\% RADIAL HEIGHT. EVERY 2ND GRIDLINE IS SHOWN.

the blade surface the nondimensional wall distance is $y^{+} \approx 1$. At 
the blade tips, casing and hub, $y^{+}$was kept at a value of $y^{+} \approx 30$. Thus, a low-Reynolds boundary condition could be chosen for the blade surfaces, while wall functions were applied on blade tips, hub and casing walls.

\section{UNSTEADY EFFECTS}

The unsteady simulations presented in this study were based on a single-frequency phase-lag approach. Therefore it was possible to compute only one passage per blade row taking into account one single blade row interaction. A full annulus simulation for the whole machine would lead to drastic CPU and memory requirements. In the narrow sense the URANS simulations are therefore combined RANS-URANS simulations where only one interaction is computed in a time-accurate way and the rest of the machine is coupled by an unsteady mixing-plane and simulated by a RANS method. For that reason, a primary analysis about the impact of the particular blade row effects is needed. In order to ensure that unsteady effects are not misinterpreted as effects due to the formulation of boundary conditions, all URANSsimulations were conducted with unsteady boundary conditions as well as with steady boundary conditions. Figure 3 shows exemplarily the comparison of the distributions of the static pressure coefficient at midspan of the first rotor blade at ADP. There is no noticeable difference, so effects of the boundary conditions can be excluded.

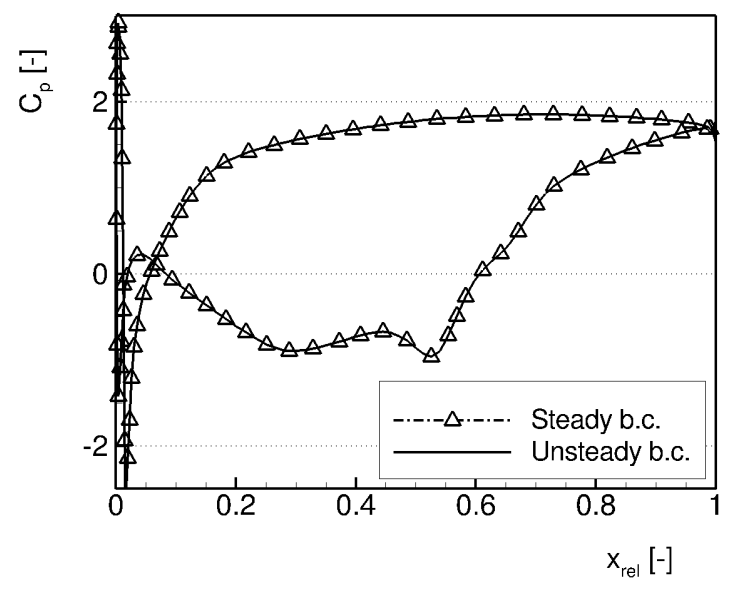

FIGURE 3. INFLUENCE OF DIFFERENT BOUNDARY CONDITIONS ON THE ROTOR BLADE PRESSURE DISTRIBUTION AT $50 \%$ RADIAL HEIGHT (ADP)

\section{Rotor-stator interaction}

Figure 4 shows a comparison between the averaged unsteady solution of the interaction between rotor 1 and stator 1 with the

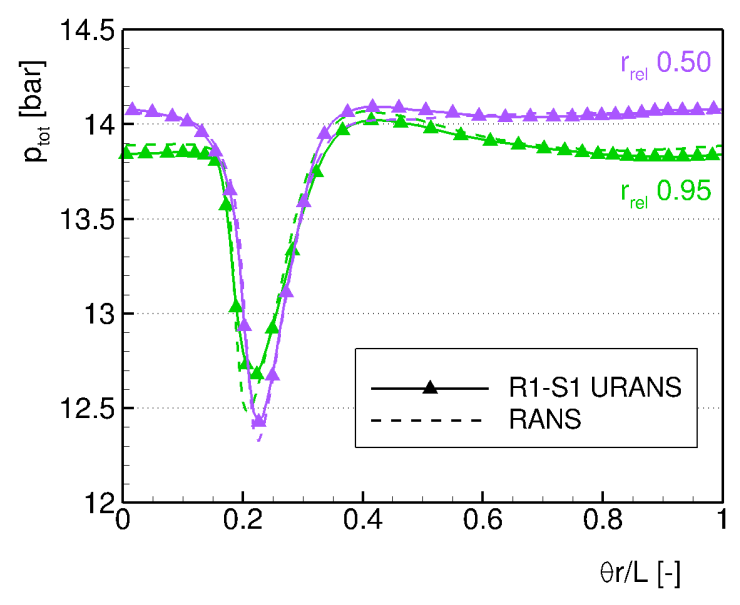

FIGURE 4. CIRCUMFERENTIAL DISTRIBUTION OF TOTAL PRESSURE AT THE EXIT OF STATOR 1 AT 50\% AND 95\% RADIAL HEIGHT (ADP)

steady result for $50 \%$ and $95 \%$ span. Both modeling strategies yield almost the same wake shape at ADP flow conditions. At 95\% span, the URANS approach predicts a slightly larger velocity deficit which has no significant effect on the downstream flow. This is an exemplary comparison representing all remaining rotor-stator interactions within this machine.

\section{Stator-rotor interaction}

Figure 5 illustrates the conducted time-accurate stator-rotor simulations. Figure 6 shows the performance comparison be-

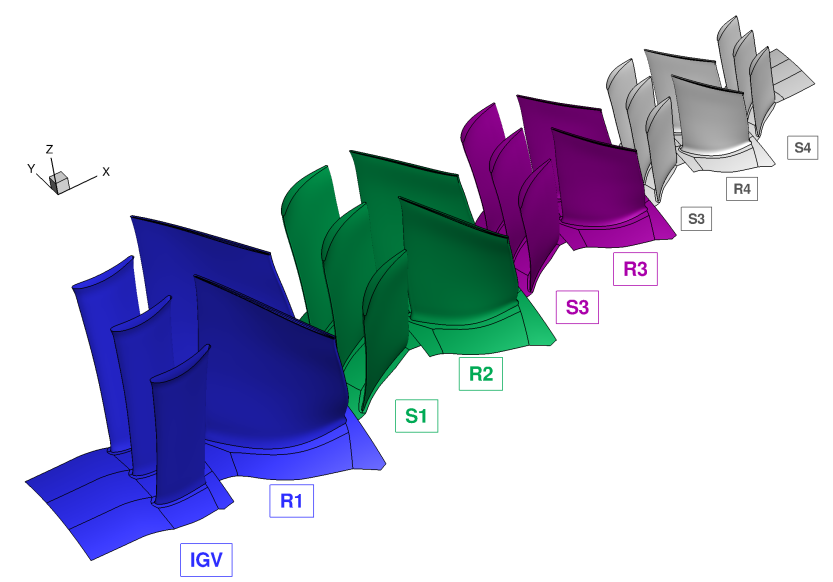

FIGURE 5. ILLUSTRATION OF THE CONDUCTED TIMEACCURATE SIMULATIONS tween experiment, steady and the unsteady simulations of all 


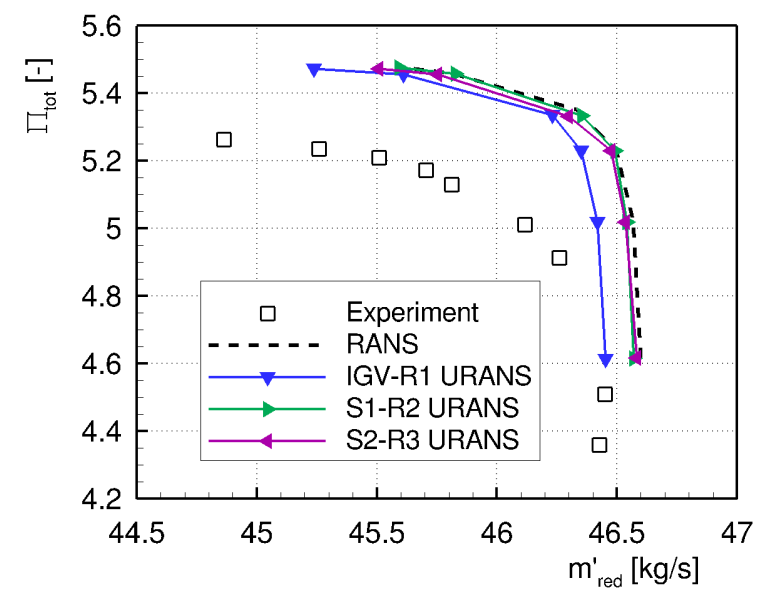

FIGURE 6. PERFORMANCE COMPARISON BETWEEN EXPERIMENT, RANS- AND URANS SIMULATION

stator-rotor interactions at design speed. At first sight there is a

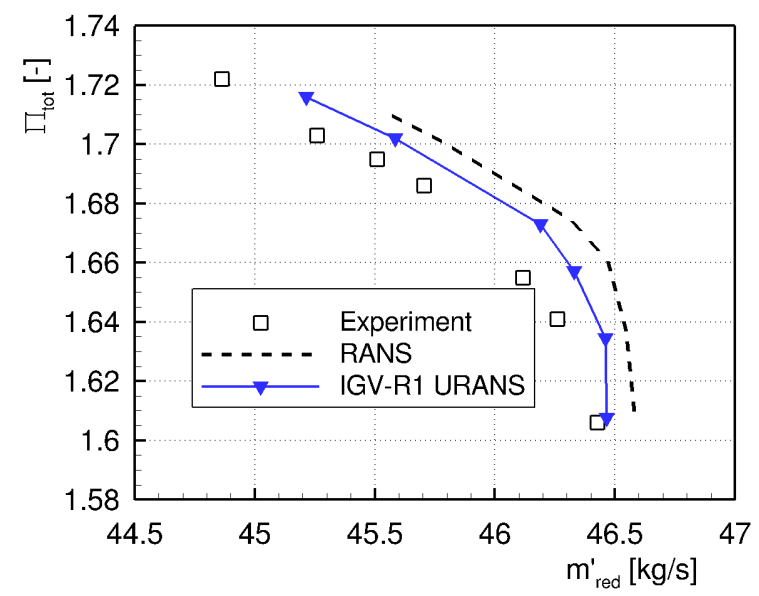

FIGURE 7. PERFORMANCE COMPARISON BETWEEN EXPERIMENT, RANS- AND URANS SIMULATION FOR THE FIRST STAGE

considerable difference between measured and computed results. The experimental total pressure ratio lies below the simulated solution over the whole operating range. This behaviour can be attributed to the k- $\omega$ turbulence model in TRACE which, in conjunction with a low-Reynolds modeling on the blade surfaces, always overpredicts the flow deviation leading to a stronger deceleration of the flow and therefore to a higher total pressure ratio. Nevertheless the interaction between IGV and rotor 1 has a strong impact on the speedline of the machine regarding the prediction of the stability limits while the unsteady interaction between the first two stators and their respective adjacent rotors has no significant influence on the machine performance. This study therefore focuses on the unsteady interaction between the IGV and rotor 1.

Figure 7 compares the experimental results with the steady and the unsteady solution for the first stage. The unsteady solution lies notably closer to the experimental result than the steady solution with regard to the absolute total pressure and the operating range.

Physical interpretation. The rotor shock has a significant impact on the upstream flowfield. Figure 8 illustrates the physical interaction mechanism. It shows the axial gap region

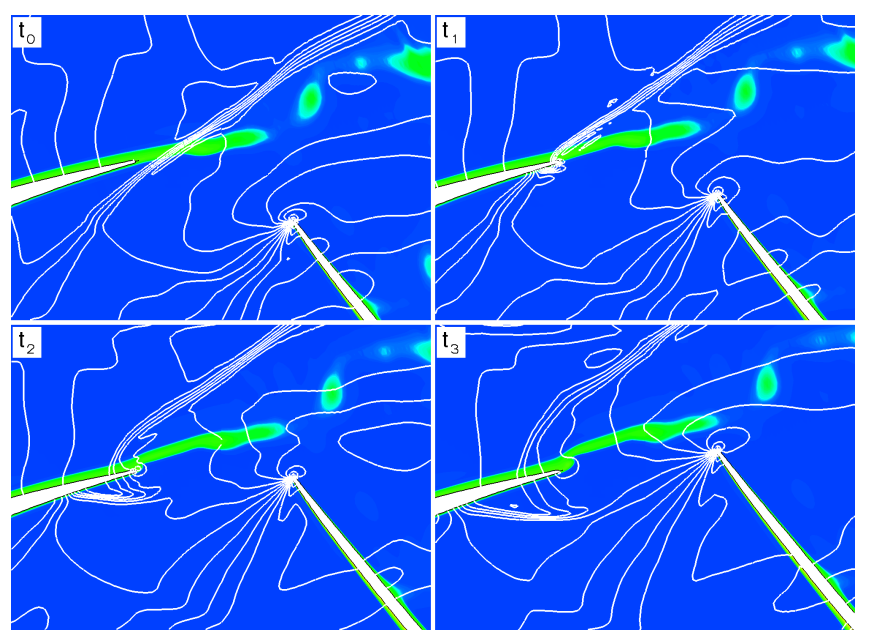

FIGURE 8. TIME VARYING ENTROPY FIELD WITH ISOBARS AT FOUR DIFFERENT TIMESTEPS

between a fixed stator blade and a rotor blade of a transonic stator-rotor configuration. The rotor moves upwards during the picture series. The illustrated values are the entropy contours and isobars (white lines). The regions of high entropy (green zones) represent the vortices and their generation. The domains of bundled isobars indicate the rotor bow shock. At timestep $t_{0}$ the shock wave approaches the trailing edge of the stator where it impinges at timestep $t_{1}$. The shock wave is reflected on the pressure side $\left(t_{2}\right)$ which generates a significant pressure rise behind the reflected shock segment. This region of high pressure causes the flow to accelerate around the trailing edge to the lower pressure region on the suction surface. Since the stator flow field is subsonic, a pressure wave is generated at the trailing edge to equalize the pressure on the upper and on the lower surfaces. As this wave is established, the stagnation point moves along the trailing edge and the pressure surface acceleration region is diminished [19]. The stagnation point therefore moves periodically up and down which drives a vortex formation and consequently a determinis- 
tic stress production. The frequency of the vortex shedding is synchronised with the rotor blade passing frequency.

Figure 9 shows the radial distribution of the total pressure behind the IGV for an operating point near the choke limit. The

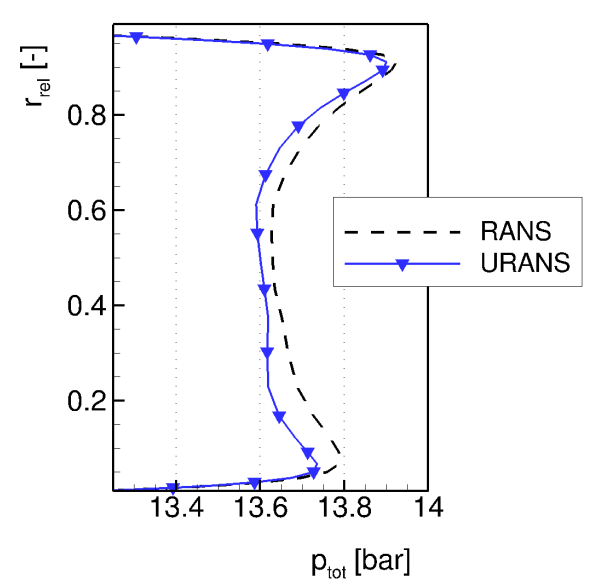

FIGURE 9. RADIAL DISTRIBUTION OF TOTAL PRESSURE BEHIND THE IGV

unsteady interaction produces a change in the wake contour, an increase of boundary layer thickness and a modification in flow deviation. The rotor is therefore subjected to another operating point and thus to a change in incidence. Figure 10 shows the tangential flow angle calculated downstream of the interface between the IGV and the first rotor. At $30 \%$ radial height there is

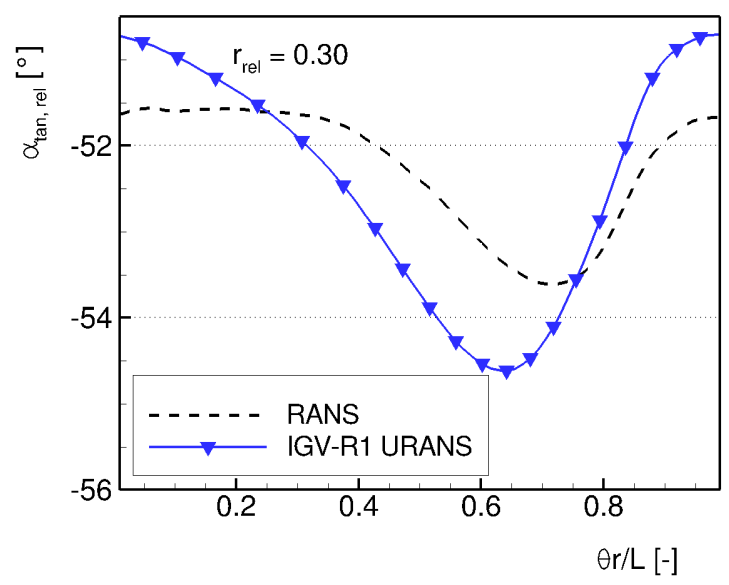

FIGURE 10. RELATIVE TANGENTIAL INCIDENCE OF ROTOR 1 AT $30 \%$ RADIAL HEIGHT

an absolute difference of up to $1.5^{\circ}$ over the whole pitch. The averaged unsteady tangential angle of incidence in the wake region is clearly smaller than the steady angle. In the outer wake region, the opposite is true.

This change in incidence leads to different pressure distributions

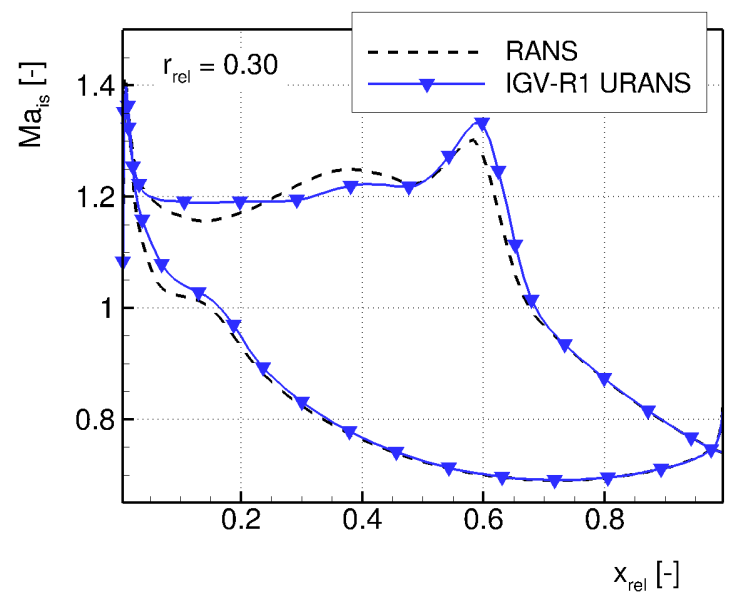

FIGURE 11. ISENTROPIC MACH NUMBER OVER ROTOR 1 AT $30 \%$ RADIAL HEIGHT NEAR CHOKE

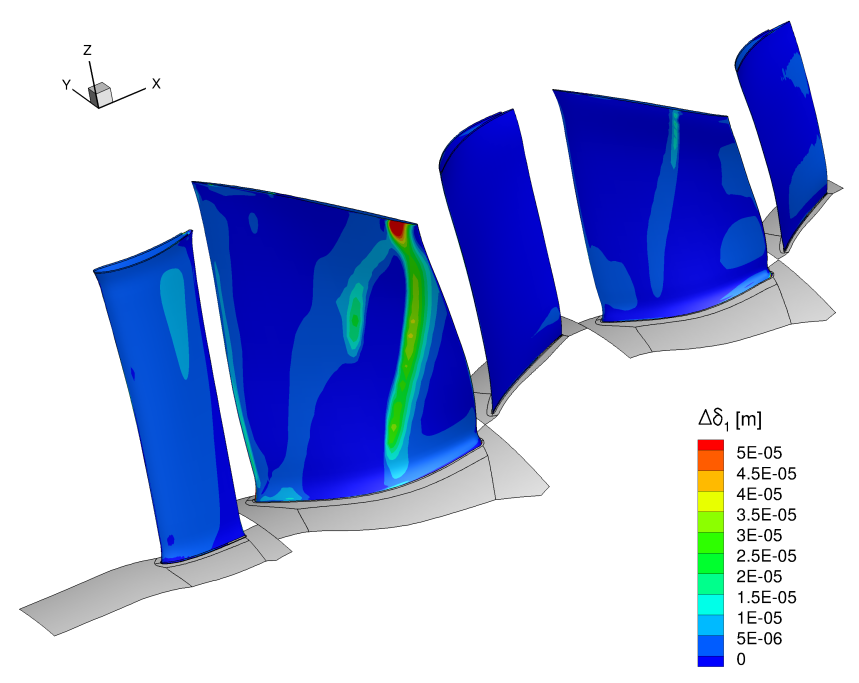

FIGURE 12. DIFFERENCE BETWEEN UNSTEADY AND STEADY DISPLACEMENT THICKNESS

on the rotor blade and therefore to a higher pre shock mach number (cf. fig. 11). Consequently the intensity of the shock boundary layer interaction becomes stronger and effects an increase of boundary layer thickness. Figure 12 illustrates this using the 
difference of time averaged unsteady and steady displacement thickness $\Delta \delta_{1}=\delta_{1}^{U R A N S}-\delta_{1}^{R A N S}$. The region of increased displacement thickness is located immediately behind the shock on the suction side and causes a reduction of the flow area. This leads to the shift of the operating range (see figs. 6 and 7).

\section{MODEL RESULTS}

In Fig. 13, the data from Fig. 6 is compared to the results of the deterministic flux model (RANS+DF) and the transport model for deterministic stresses (RANS+DS). The flux model captures the massflow displacement and produces only a slight improvement of the total pressure ratio. This is due to the fact, that the unsteady and the steady boundary conditions are not fully consistent. In order to optimize the deterministic flux approach, the unsteady nonreflecting boundary conditions will be revised in future work. Currently they use a simplification proposed by Giles [20] ensuring that the boundary conditions are local in time and space. Therefore the unsteady boundary conditions represent only a second order approximation, whilst the steady boundary conditions are exact for two-dimensional Euler flows. However, the transport model shows a better performance. The total pressure ratio is nearly identical with the unsteady reference solution and in particularly is able to predict operating points near the surge limit which is a considerable benefit compared to the standard steady approach where the last converged operating point lies at a reduced massflow of $\dot{m}_{\text {red }} \approx 45,6 \mathrm{~kg} / \mathrm{s}$. The capabil-

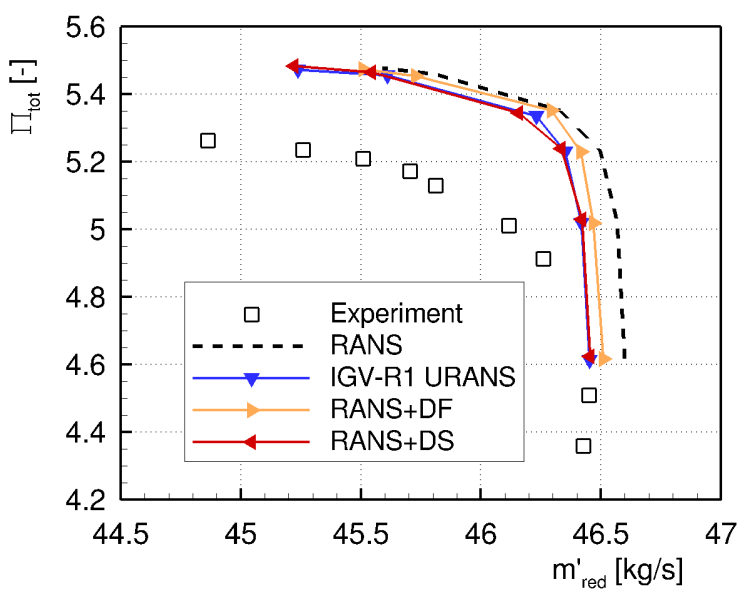

FIGURE 13. COMPARISON BETWEEN EXPERIMENT AND DIFFERENT SIMULATION STRATEGIES FOR THE WHOLE COMPRESSOR AT DESIGN SPEED

ity of both deterministic modeling approaches is also apparent in Fig. 14 where the speedline for the first stage is plotted (cf. fig. 7). The deterministic flux model shows a slight improvement of

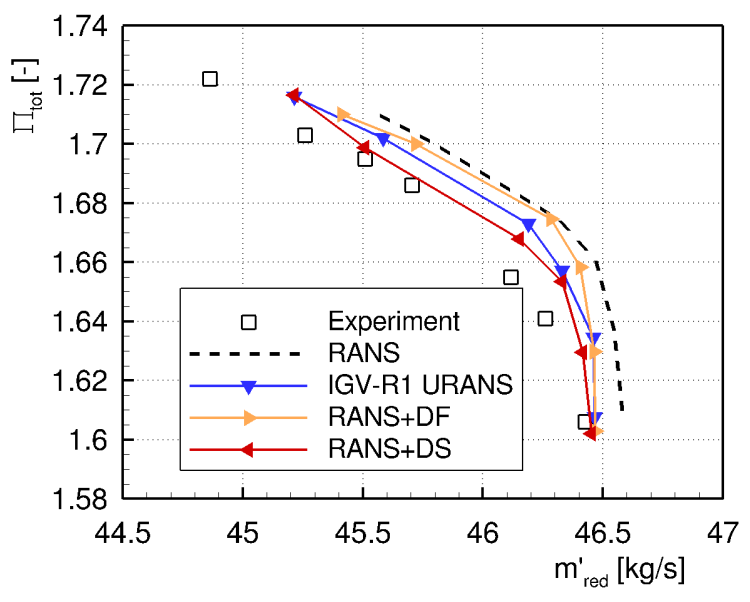

FIGURE 14. COMPARISON BETWEEN EXPERIMENT AND DIFFERENT SIMULATION STRATEGIES FOR THE FIRST STAGE AT DESIGN SPEED

the steady approach whereas the transport model is even closer to the experiment than the unsteady solution.

Beside the prediction quality the runtime of the transport model is beneficial. While the unsteady simulations take about 17 times longer than the steady simulations, the DS-model runtime exceeds the steady one only about $7 \%$.

\section{Comparison of modeling strategies at constant mass- flow}

Figures 15 - 17 show a direct comparison of the different modeling strategies at a massflow of $\dot{m}_{\text {red }} \approx 46,3 \mathrm{~kg} / \mathrm{s}$. In Fig.

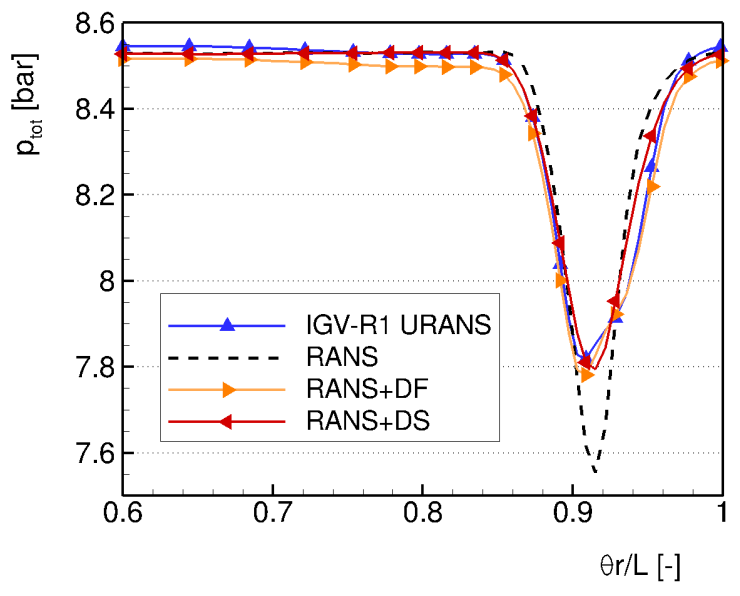

FIGURE 15. CIRCUMFERENTIAL DISTRIBUTION OF TOTAL PRESSURE AT THE EXIT OF THE IGV AT MIDSPAN 
15 the circumferential distributions of the total pressure is depicted at the exit of the IGV at midspan. The wake shape is modified because of the unsteady interaction. The wake shape is expanded and bares a strongly reduced velocity deficit. Both effects are captured by the deterministic models.

Hence, the improved flowfield is also visible in the static pressure and isentropic Mach number distribution around the rotor blade profile (fig. 16). The deterministic models are able to correct the shock position compared to the steady approach. As experimen-

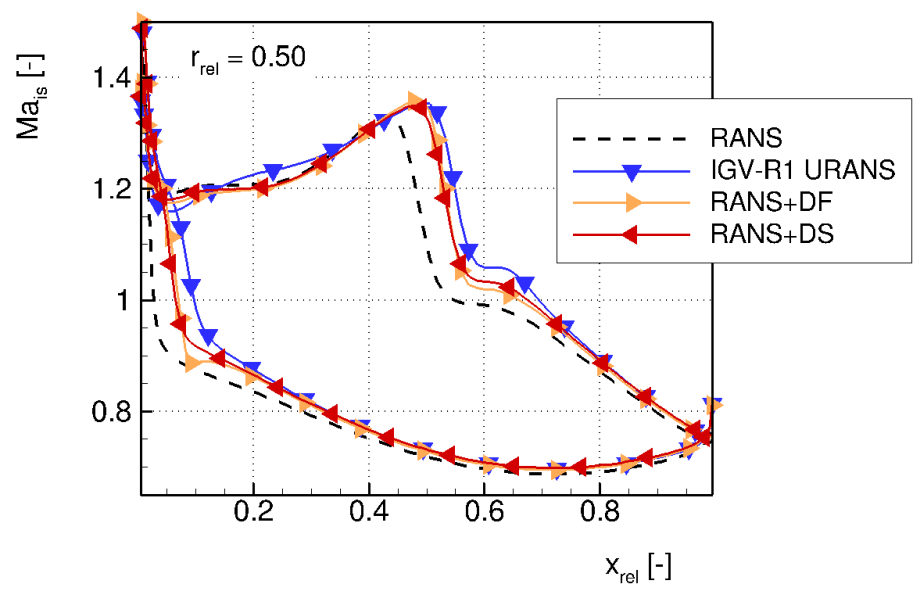

FIGURE 16. ISENTROPIC MACH NUMBER OVER ROTOR 1 AT MIDSPAN

tal data at the stator leading edges is available, Fig. 17 compares the simulation solutions to the experimental data at that location. The deterministic models improve the steady solution clearly towards the unsteady and the experimental results.

\section{CONCLUSIONS}

In this paper two different modeling strategies for deterministic stresses were presented and applied on a transonic compressor. The model results showed an improved prediction of performance data, radial distributions, blade loadings and other relevant flow data. The deterministic flux approach shows good agreement with the reference data but still has room for improvement regarding the unsteady boundary conditions as mentioned above. The transport model for deterministic stresses demonstrates the ability to further improve the conventional steady mixing-plane approach used for design purposes as its runtime exceeds the steady runtime about only $7 \%$. Beyond that, it is able to predict operating points that are located much closer to the surge limit.

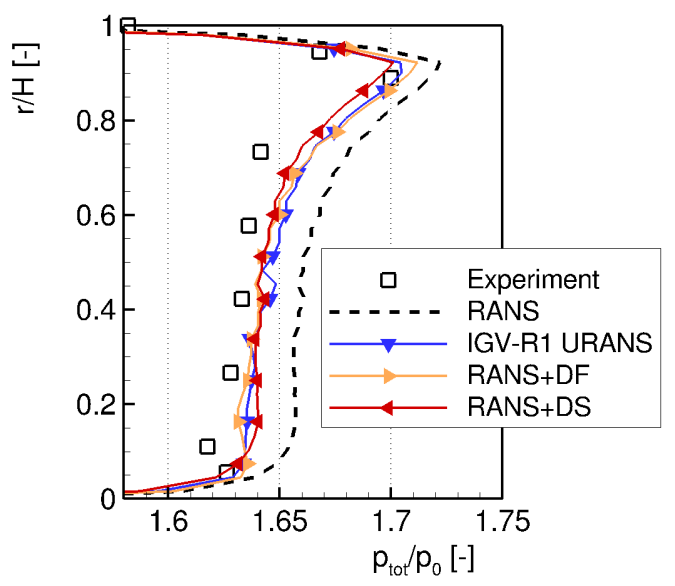

FIGURE 17. RADIAL DISTRIBUTION OF TOTAL PRESSURE IN FRONT OF STATOR 1

\section{REFERENCES}

[1] Greitzer, E., Tan, C., Wisler, D., Adamczyk, J., and Strazisar, A., 1994. "Unsteady flow in turbomachines: Where's the beef?". Unsteady Flows in Aeropropulsion, 40, pp. 1-11.

[2] Trebinjac, I., Charbonnier, D., and Leboeuf, F., 2005. "Unsteady rotor-stator interaction in high speed compressor and turbine stages". J. of Thermal Science, 14(4).

[3] Adamczyk, J., 1985. "Model equation for simulating flows in multistage turbomachinery". ASME Paper 85-GT-226.

[4] Hall, E., 1997. "Aerodynamic modeling of multistage compressor flowfields - part 2: Modeling deterministic stresses". ASME Paper 97-GT-345.

[5] Rhie, C., Gleixner, A., Spear, D., Fischberg, C., and Zacharias, R., 1995. "Development and application of a multistage navier-stokes solver - part i: Multistage modeling using bodyforces". ASME Paper 95-GT-342.

[6] van de Wall, A., Kadambi, J., and Adamczyk, J., 2000. "A transport model for the deterministic stresses associated with turbomachinery blade row interactions". ASME Journal of Turbomachinery, 122, pp. 593-603.

[7] Charbonnier, D., and Leboeuf, F., 2004. "Steady flow simulation of rotor-stator interactions with a new unsteady flow model". AIAA Paper 2004-3754.

[8] Hall, K., Thomas, J., and Clark, W., 2002. "Computation of unsteady nonlinear flows in cascades using a harmonic balance technique". AIAA Journal, 40 No. 5, pp. 879-886.

[9] He, L., and Ning, W., 1998. "Efficient approach for analysis of unsteady viscous flows in turbomachines". AIAA Journal, 36 No. 11, pp. 2005-2012.

[10] Sondak, D., Dorney, D., and Davis, R., 1996. "Modeling turbomachinery unsteadiness with lumped deterministic stresses”. AIAA Paper 96-2570. 
[11] Lukovic, B., Orkwis, P., Turner, M., and Sekar, B., 2006. "Neural network modeling of deterministic unsteadiness source terms". AIAA Journal, 44, No.7, pp. 1609-1616.

[12] Stollenwerk, S., and Kügeler, E., 2011. "Unsteady flow modelling using deterministic flux terms". Proceedings of the 9th European Conference on Turbomachinery, Fluid Dynamics and Thermodynamics, Istanbul, Turkey, pp. 759768.

[13] Stollenwerk, S., and Nürnberger, D., 2009. "A model for potential deterministic effects in transonic compressor flows". ASME Paper GT-2009-60105.

[14] Becker, K., Heitkamp, K., and Kügeler, E., 2011. "Recent progress in a hybrid-grid cfd solver for turbomachinery flows". Proceedings of the 5th European Conference on Computational Fluid Dynamics, ECCOMAS CFD, Lissabon, Portugal.

[15] Stollenwerk, S., and Nürnberger, D., 2008. "A model for potential deterministic effects in turbomachinery flows". Proceedings of ICME 2008, London, U.K., pp. 1386-1391.

[16] Röber, T., Kügeler, E., and Weber, A., 2010. "Investigation of unsteady flow effects in an axial compressor based on whole annulus computations". ASME Paper GT201023522.

[17] Johann, E., and Heinichen, F., 2011. "Back to back comparison of a casing treatment in a high speed multi-stage compressor rig test". ISABE-Paper 2011-1223.

[18] Weber, A., 2009. "G3dmesh - 3d structured grids for multistage / multipassage turbomachines and linear cascades". Institutsbericht DLR IB 325-06-09.

[19] Sanders, A., and Fleeter, S., 1999. "Transonic rotor-igv interactions". ISABE Paper 99-7029.

[20] Giles, M., 1991. "Unsflo: A numerical method for the calculation of unsteady flow in turbomachinery". GTL-Report, MIT Gas Turbine Laboratory, 205. 